\title{
Pyruvate oxidase of Streptococcus pneumoniae contributes to pneumolysin release
}

\author{
Joseph C. Bryant ${ }^{1}$, Ridge C. Dabbs ${ }^{1}$, Katie L. Oswalt', Lindsey R. Brown¹, Jason W. Rosch², Keun S. Seo ${ }^{3}$,
} Janet R. Donaldson ${ }^{4}$, Larry S. McDaniel ${ }^{5}$ and Justin A. Thornton ${ }^{1 *}$

\begin{abstract}
Background: Streptococcus pneumoniae is one of the leading causes of community acquired pneumonia and acute otitis media. Certain aspects of S. pneumoniae's virulence are dependent upon expression and release of the protein toxin pneumolysin (PLY) and upon the activity of the peroxide-producing enzyme, pyruvate oxidase (SpxB). We investigated the possible synergy of these two proteins and identified that release of PLY is enhanced by expression of SpxB prior to stationary phase growth.
\end{abstract}

Results: Mutants lacking the spxB gene were defective in PLY release and complementation of spxB restored PLY release. This was demonstrated by cytotoxic effects of sterile filtered supernatants upon epithelial cells and red blood cells. Additionally, peroxide production appeared to contribute to the mechanism of PLY release since a significant correlation was found between peroxide production and PLY release among a panel of clinical isolates. Exogenous addition of $\mathrm{H}_{2} \mathrm{O}_{2}$ failed to induce PLY release and catalase supplementation prevented PLY release in some strains, indicating peroxide may exert its effect intracellularly or in a strain-dependent manner. SpxB expression did not trigger bacterial cell death or LytA-dependent autolysis, but did predispose cells to deoxycholate lysis.

Conclusions: Here we demonstrate a novel link between spxB expression and PLY release. These findings link liberation of PLY toxin to oxygen availability and pneumococcal metabolism.

Keywords: Streptococcus pneumoniae, Pneumococcus, Pneumolysin, Virulence, Toxin, Metabolism, Protein secretion, Cytotoxicity

\section{Background}

S. pneumoniae (pneumococcus) is a Gram-positive human pathogen identified as a cause of acute otitis media, bacteremia, septicemia, pneumonia, and meningitis [1] and is the leading cause of death in children under the age of five worldwide [2]. Diseases resulting from pneumococcal infection impart a major economic impact, with healthcare costs estimated to range from 3 billion to 6 billion dollars annually in the United States for otitis alone [3]. In spite of this major disease burden, it most often exists as a commensal organism of the

\footnotetext{
* Correspondence: thornton@biology.msstate.edu

'Department of Biological Sciences, Mississippi State University, 295 E Lee

Blvd., Harned Hall, Rm 219, Mississippi State, MS 39762, USA

Full list of author information is available at the end of the article
}

nasopharynx, with carriage rates of up to $70 \%$ depending on the demographic [4]. Pneumococcus produces and secretes a number of surface proteins which contribute to virulence including neuraminidase, hyaluronidase, and pneumococcal surface protein A (PspA) [5-7]. However, it produces relatively few protein exotoxins compared to other pathogenic species capable of such invasive disease.

The primary toxin expressed by S. pneumoniae is pneumolysin (PLY), which is a $53-\mathrm{kDa}$ cholesterol dependent pore-forming cytolysin (CDC) [8]. PLY has been found to reduce ciliary beating within the lungs and deletion of the ply gene from the pneumococcal chromosome attenuates virulence in vivo [9]. Additionally, exposure to PLY activates differential gene expression within host cells [10]. 
Unlike the other members of the CDC family, PLY lacks an $\mathrm{N}$-terminal signal sequence for extracellular release via the Sec-dependent pathway [11]. Despite this, it is well established that PLY is released into the extracellular space $[8,9,12]$.

Controversy exists as to the primary route PLY takes to exit the cytoplasm. The mechanism of release was long thought to be solely attributable to autolysis of the bacterial cell [13]. However, PLY release has been demonstrated in the absence of autolysis [14], suggesting that other mechanisms must contribute to liberation of PLY during pneumococcal growth. Other findings have demonstrated that PLY can actually traverse and associate with the pneumococcal cell wall [15]. Subsequent studies by Price et al. demonstrated that domain 2 of PLY is essential for the cell wall association and that the export pathway was conserved in Bacillus subtilis [12]. The composition of the pneumococcal peptidoglycan has been recently shown to restrict PLY release [16]. Interestingly, this study found that greater PLY release does not correlate with enhanced virulence and that rather a controlled release of PLY is important for pathogenesis. These findings underline the fact that, while capable of inflicting damage to the host, PLY is also a stimulator of host immune responses [17].

S. pneumoniae is unique among catalase-negative organisms due to the fact that it produces up to millimolar concentrations of hydrogen peroxide $\left(\mathrm{H}_{2} \mathrm{O}_{2}\right)$, primarily through the activity of the enzyme pyruvate oxidase $(\mathrm{SpxB})[18,19]$. In aerobic environments, pneumococcus utilizes $\mathrm{SpxB}$ to convert pyruvate to acetate, a reaction that produces acetyl phosphate, $\mathrm{CO}_{2}$, and $\mathrm{H}_{2} \mathrm{O}_{2}$ [20]. Although SpxB-derived $\mathrm{H}_{2} \mathrm{O}_{2}$ is detrimental to survival of the pneumococcus at high concentrations, deletion of $\operatorname{sp} x B$ has been shown to reduce virulence in vivo [21]. Pneumococcal $\mathrm{H}_{2} \mathrm{O}_{2}$ has also been shown to aid the pneumococcus in competing with other inhabitants of the upper respiratory tract [22] and possibly has a significant impact upon host cells and tissues. It is cytotoxic to numerous cell types including neuronal cells, neutrophils, and alveolar epithelial cells [23-26]. During stationary phase, pneumococcal $\mathrm{H}_{2} \mathrm{O}_{2}$ results in pneumococcal cell death resembling apoptosis of eukaryotic cells and this process does not require the major autolysin, $\mathrm{N}$-acetylmuramoyl-l-alanine amidase (LytA) [27]. However, strains vary in their production of $\mathrm{H}_{2} \mathrm{O}_{2}$, with some strains producing significant concentrations prior to stationary phase. The impact of low and intermediate levels of $\mathrm{H}_{2} \mathrm{O}_{2}$ upon the physiology and structural integrity of $S$. pneumoniae has not been investigated in detail. Price et al. demonstrated PLY associating with the cell wall [15], indicating that by some mechanism PLY escapes the cell membrane. Additionally, pneumococcus is known to alter its membrane composition in response to endogenous reactive oxygen species [28] Based on these results, we hypothesized that pneumococcal $\mathrm{H}_{2} \mathrm{O}_{2}$ might have non-lethal effects on the physiology of the bacterium at early phases of growth, possibly affecting release of PLY. We investigated the impact of SpxB on PLY localization into bacterial supernatants and the effect of this upon host cell integrity.

\section{Methods}

\section{Bacterial strains and culture conditions}

S. pneumoniae strains TIGR4 [29], an unencapsulated mutant of TIGR4 (T4R) [30], WU2 [31], AW267, along with isogenic mutants of these strains were grown in Todd Hewitt media plus $0.5 \%$ yeast extract (THY) to a mid-logarithmic phase of growth $\left(\mathrm{OD}_{600}\right.$ of 0.5$)$ at $37^{\circ} \mathrm{C}$. Clinical isolates were received from the Center for Disease Control and Prevention's Active Bacterial Core surveillance (ABCs) isolate bank (http://www.cdc.gov/ abcs/index.html). For additional studies strains lacking the major cell wall amidase LytA $(\Delta l y t A)$ were created as described below. The $\triangle l y t A$ isogenic mutants of T4R and WU2, along with $\triangle l y t A \Delta s p x B$ double mutants were cultured under the same conditions as parental strains. To neutralize pneumococcal $\mathrm{H}_{2} \mathrm{O}_{2}$ from supernatants, THY media was supplemented with catalase derived from Aspergillus niger ( $2 \mathrm{ng} / \mathrm{mL}$; cat \#C3515 Sigma Aldrich). A549 type 2 lung epithelial cells (ATCC) were cultured in F12-K medium (ATCC) supplemented with $10 \%$ fetal bovine serum (FBS) at $37{ }^{\circ} \mathrm{C}$ in $5 \% \mathrm{CO}_{2}$ atmosphere.

\section{Mutant construction}

Isogenic $\triangle s p x B$ mutants were developed in the strains TIGR4, WU2, T4R, and AW267 by allelic replacement. Briefly, the $\operatorname{spxB}$ gene with 500 base pairs flanking each end was amplified from T4R chromosomal DNA using primers ALR1 and ALR2, designed to contain both KpnI and $\mathrm{XbaI}$ restriction sites. The product was digested with $\mathrm{KpnI}$ and $\mathrm{XbaI}$ restriction endonucleases (New England BioLabs, Ipswich, MA) and ligated into $\mathrm{KpnI}$ and $\mathrm{XbaI}$ digested pBluescript vector using T4 DNA ligase (Thermo Fisher Scientific). Inverse PCR was performed using primers ALR3 and ALR4 to amplify outward from just inside the coding sequence of $s p x B$ from a positive clone. Primers ALR3 and ALR4 were designed to include BamHI sites. The gene ermB was amplified using designed primers ALR5 and ALR6 including BamHI sites and both products were digested with BamHI (New England BioLabs, Ipswich, MA) overnight at $37{ }^{\circ} \mathrm{C}$. The linearized vector that was amplified with primers ALR3 and ALR4 and the ermB insert amplified with primers ALR5 and ALR6 were ligated and transformed into DH5 $\alpha$ E. coli cells. Positive colonies with bands of the 
appropriate size were grown in LB medium overnight and frozen at $-80{ }^{\circ} \mathrm{C}$. PCR products containing the knockout construct were amplified from positive clones and used to transform pneumococcal strains by standard methods and subsequent selection for transformants by plating on blood agar plates containing erythromycin $(0.5 \mu \mathrm{g} / \mathrm{mL})$. Mutants lacking the $l y t A$ gene were generated in strains T4R and WU2 by overlap extension PCR mutagenesis as previously described [32]. Briefly, 5001000 base pair DNA sequences flanking each side of the lytA gene were amplified using primers LytA-KO1, LytASup LytASdn, and LytA-KO4 (Table 1) and fused by PCR to the spectinomycin resistance cassette amplified from the shuttle vector pNE-1. The lytA gene was replaced with a spectinomycin cassette following transformation of $S$. pneumoniae strains by standard methods and selected for by plating on blood agar plates supplemented with spectinomycin $(500 \mu \mathrm{g} / \mathrm{mL})$. The T4R $\Delta p l y$ mutant was created using the same method (with primers PlyAF, PlyAR, PlyBF, PlyBR), but replacing the ply gene with an erythromycin cassette. Primer sequences for all mutants are listed in Table 1. Complemented mutants in strains TIGR4 and T4R were developed through cloning the $s p x B$ gene from T4R by

Table 1 Primer sequences

\begin{tabular}{|c|c|}
\hline Primer name & Sequence $\left(5^{\prime}-3^{\prime}\right)$ \\
\hline LytA-KO1 & GCGGGTACCCAGTCCAGCTTTGGTTTCCT \\
\hline LytASup & TAAAAATATCTCTTGCCAGTCCTTGCCTATATGGTTGCACG \\
\hline LytASdn & $\begin{array}{l}\text { GGTAATCAGATTTAAGAAAACAATAAACCCTCACAGTAGA } \\
\text { GCCAGAT }\end{array}$ \\
\hline LytA-KO4 & CGCGGATCCTCACAGTAGAGCCAGATGGC \\
\hline PlyAF & CTCAATCCAGCTACCTGTCGC \\
\hline PlyAR & GTTTGCTTCTAAGTCTTATTTCCCTTCTACCTCCTAATAAG \\
\hline PlyBF & GAGTCGCTTITGTAAATTTGGGAGAGGAGAATGCTTGCG \\
\hline PlyBR & GCTTGTTTAGCACGGTCG \\
\hline ALR1 & GCG GGTACCGCGTGCTATTGCAGATCAAA \\
\hline ALR2 & GCGTCTAGACATCGTTAATCGGAGATGGA \\
\hline ALR3 & CGCGGATCCATCTACGCCCCATGTTTCAATACG \\
\hline ALR4 & CGCGGATCCACCATTCCGTCTCTTCTTGG \\
\hline ALR5 & CGCGGATCCGGAAATAAGACTTAGAAGCAAAC \\
\hline ALR6 & CGCGGATCCCCAAATTTACAAAAGCGACTC \\
\hline SpxB-F & CGCGCCCGGGTGACAACACTTTCAAAACTG \\
\hline SpxB-R & CGCGGAATTCTTATTTAATTGCGCGTGATTGC \\
\hline ERM-F & GGAAATAAGACTTAGAAGCAAAC \\
\hline ERM-R & CCAAATTTACAAAAGCGACTC \\
\hline Spec-F & CGTGACTGGCAAGAGATATTTTA \\
\hline Spec-R & GGGTTATTGTITCTAAAATCTGATTACC \\
\hline PLY-F & CAGAGCGTCCTTTGGTCTATATT \\
\hline PLY-R & CAGCCTCTACTTCATCACTCTTAC \\
\hline
\end{tabular}

amplifying the gene by PCR with primers SpxB-F and SpxB-R followed by digestion of the product with EcoRI and XmaI enzymes (New England Biolabs, Ipswich, MA). Following digestion of the product, the gene was ligated with the pNE-1 pneumococcal shuttle vector that was similarly digested. The ligation was transformed into E. coli strain DH5 $\alpha$. Purified plasmid was then used to transform $\triangle s p x B$ strains by standard methods and complemented mutants were selected by plating on blood agar plates supplemented with erythromycin $(0.5 \mu \mathrm{g} / \mathrm{mL})$ and spectinomycin $(500 \mu \mathrm{g} / \mathrm{mL})$. Complementation of $s p x B$ was confirmed by PCR and by assaying supernatant from clones by a Pierce Quantitative Peroxide Assay (cat\# 23280; Life Technologies).

\section{$\mathrm{H}_{2} \mathrm{O}_{2}$ quantitation}

Pneumococci were grown to mid-log phase $\left(\mathrm{OD}_{600}\right.$ of $0.5)$. Following centrifugation $(16,000 \times \mathrm{g}$ for $5 \mathrm{~min})$ of $1 \mathrm{~mL}$ of the culture, the supernatant was subsequently filtered through a $0.22 \mu \mathrm{m}$ polyethersulfone (PES) membrane syringe filter (CellTreat) to remove remaining bacterial cells and analyzed for hydrogen peroxide production using a colorimetric hydrogen peroxide quantification assay per manufacturer instructions in a BioTek Synergy HT plate reader at an $\mathrm{OD}_{540}$ (cat\# 23280; Life Technologies). This analysis was performed in triplicate.

\section{$\mathrm{H}_{2} \mathrm{O}_{2}$ Treatment}

To ascertain the impact of $\mathrm{H}_{2} \mathrm{O}_{2}$ alone on the release of PLY, T4R and its isogenic $\triangle s p x B$ mutant were grown to mid $\log$ phase $\left(\mathrm{OD}_{600}\right.$ of 0.5$)$ in $5 \mathrm{~mL}$ of THY media and treated with either $0 \mu \mathrm{M}$ or $500 \mu \mathrm{M} \mathrm{H}_{2} \mathrm{O}_{2}$. Samples were then placed on ice for $1 \mathrm{~h}$, after which $\mathrm{H}_{2} \mathrm{O}_{2}$ was quantified following centrifugation of $1 \mathrm{~mL}$ of culture at $15,000 \mathrm{rpm}$ for $5 \mathrm{~min}$ and filtration of the supernatant through PES (CellTreat) syringe filters as described above. Bacterial counts were determined before and after the $\mathrm{H}_{2} \mathrm{O}_{2}$ treatment by viable plate counts using blood agar plates.

\section{Western blot}

To determine the relative amount of PLY released between parental and mutant strains, sterile-filtered bacterial supernatants from cultures grown to mid-log phase $\left(\mathrm{OD}_{600}\right.$ of 0.5$)$ were denatured by boiling for 5 min and separated on $10 \%$ SDS-PAGE gels (Bio-Rad) prior to being transferred onto a $0.22 \mathrm{~nm}$ PVDF membrane (Millipore). The membranes were then blocked for $30 \mathrm{~min}$ (5\% milk) and probed with rabbit polyclonal anti-PLY antibody overnight. The blot was subsequently washed with and probed with goat anti-rabbit HRP conjugated secondary antibody (BioRad) for $1 \mathrm{~h}$. Blots were incubated with Luminata Forte substrate (Millipore) for 
$1 \mathrm{~min}$ at $25{ }^{\circ} \mathrm{C}$. The membranes were then developed following exposure to radiography film (GeneMate). Band density was calculated using Image software after scanning film at $600 \mathrm{dpi}(\mathrm{NCBI})$.

\section{Dot blot}

To quantitate PLY in the supernatants, recombinant PLY was serially diluted into THY media to create a standard of known concentration (20 ng to $5 \mu \mathrm{g}$ ). PLY standards $(20 \mu \mathrm{L})$ and bacterial supernatants $(100 \mu \mathrm{L})$ were applied onto PVDF membranes using a dot blotting vacuum manifold (Bio-Rad). Following $45 \mathrm{~min}$ of gravity filtration, light vacuum was applied to adhere the protein to the membrane and the wells were washed twice using $200 \mu \mathrm{L}$ of phosphate buffered saline (PBS). The membrane was then blocked for $30 \mathrm{~min}$ in $5 \%$ milk. The membrane was then probed with an anti-PLY rabbit polyclonal antibody at a 1:200 dilution overnight. Following exposure to the primary antibody, the blot was then probed with a goat anti-rabbit secondary for $1 \mathrm{~h}$. The blot was developed using Luminata Forte substrate (Millipore) and exposed to X-ray film (GeneMate). Dot density was quantitated using Image (NCBI) and then plotted against the known concentration of standards to determine quantity of PLY per $100 \mu \mathrm{L}$.

\section{Real time PCR}

Pneumolysin gene expression in T4R, WU2, AW267, and their respective isogenic $\triangle s p x B$ mutants was quantitated by qRT-PCR. Bacterial strains were grown to an $\mathrm{OD}_{600}$ of 0.5 in THY. Bacterial RNA was extracted following sonication of the bacterial culture, and a total of 4 min of bead beating with $0.1 \mathrm{~mm}$ zircon beads. Bacterial RNA was purified using the Qiagen RNeasy kit, with the inclusion of an on column RNase-free DNase treatment for $1 \mathrm{~h}$. Bacterial RNA was quantitated using a Qubit fluorometer and $50 \mathrm{ng}$ was used to generate cDNA utilizing a Maxima cDNA synthesis kit for qRTPCR (Life Technologies). Gene-specific primer sequences PLY-F and PLY-R are listed in Table 1. The fold-change in gene expression was calculated using the $\Delta \Delta \mathrm{C}_{\mathrm{T}}$ method utilizing $g y r A$ as an internal control.

\section{Flow cytometry}

To assess the effect of bacterial supernatants upon human cells, $1 \times 10^{5}$ A549 epithelial cells were treated with filtered bacterial supernatant obtained from T4R, $\mathrm{T} 4 \mathrm{R} \Delta s p x B$, T4R $\Delta p l y$, and the complemented T4R $\Delta s p x B$ $(\triangle s p x B+)$. Briefly, epithelial cells were pelleted by centrifugation at $380 \times \mathrm{xg}$ for $4 \mathrm{~min}$, suspended in $0.2 \mathrm{~mL}$ of filtered bacterial supernatant, and incubated at $37{ }^{\circ} \mathrm{C}$ for $30 \mathrm{~min}$ on a rotating platform. Cells were then pelleted at $380 \times \mathrm{g}$ for $4 \mathrm{~min}$ and suspended in $0.5 \mathrm{~mL}$ PBS with $3 \mu \mathrm{g} / \mathrm{mL}$ propidium iodide (PI) (Sigma Aldrich). The cells were assessed using an Attune Flow Cytometer (Life Technologies) and the fluorescence intensity shift in the BL3 channel was measured as an indicator of epithelial cell death.

\section{Hemolysis assay}

PLY release was quantitated by standard hemolytic assay. Briefly, bacterial supernatants from cultures grown to an $\mathrm{OD}_{600}$ of 0.3 were serially diluted (1:3) across the microtiter plate into phosphate-buffered saline (PBS) containing $0.1 \%$ dithiothreitol (Sigma, St. Louis, MO). Washed $1 \%$ sheep red blood cells in PBS were added to the wells and incubated at $37^{\circ} \mathrm{C}$ for $30 \mathrm{~min}$. After incubation, the sheep erythrocytes were pelleted and plates were imaged.

\section{Extracellular DNA quantitation}

Bacterial strains were grown in THY medium to an $\mathrm{OD}_{600}$ of 0.5 and extracellular DNA was quantitated from sterile filtered supernatant by a Qubit fluorometer using Qubit dsDNA HS Assay Kit (Thermo Fisher Scientific).

\section{Autolysis}

S. pneumoniae T4R and the isogenic $\triangle s p x B$ mutant were grown in $12 \mathrm{~mL}$ of $\mathrm{THY}$ at $37^{\circ} \mathrm{C}$. Upon reaching $\mathrm{OD}_{600}$ 0.45 , each $12 \mathrm{~mL}$ culture was split into three separate tubes. The separate tubes containing mid-log phase bacteria were incubated at room temperature until reaching an $\mathrm{OD}_{600}$ of 0.5 . Upon reaching the desired OD, Triton X-100 (0.5\%) or sodium deoxycholate $(0.05 \%)$ was added to the respective culture tubes and the $\mathrm{OD}_{600}$ of the cultures was recorded every minute for a total of $10 \mathrm{~min}$ for each of the three culture tubes. Controls received no detergents. Additional control and experimental samples were grown in the presence of catalase $(2 \mu \mathrm{g} / \mathrm{mL})$.

Statistical analysis. All statistical analyses (Student's $t$ test (two-tailed) and linear best-fit regression) were performed using GraphPad Prism software (www.graphpad.com) with a $p$-value $<0.5$ considered significant.

\section{Results}

\section{Expression of SpxB enhances PLY-dependent cell death}

To determine the relative contribution of $\mathrm{H}_{2} \mathrm{O}_{2}$ and PLY secreted by pneumococcus to cytolysis of airway epithelial cells, A549 human lung epithelial cells were exposed to filter-sterilized supernatants collected from mid-log phase cultures of $S$. pneumoniae and isogenic mutants lacking either $s p x B$ or ply genes and the loss of membrane integrity was assessed by uptake of PI. Compared to THY media alone, exposure to supernatant from the parental strain T4R led to a nearly complete loss of A549 cell viability (89\%), while exposure to supernatant from a strain lacking pyruvate oxidase (T4R $\Delta s p x B$ ) resulted in minimal loss in membrane integrity (1.65 \%; 
Fig. 1a). This effect was shown to be dependent upon release of PLY into the supernatant since supernatant from a strain containing a functional $\operatorname{spx} B$ gene but lacking the ply gene (T4R $\Delta p l y)$ resulted in only $2.52 \%$ of cells staining PI positive. Complementation of $s p x B$ $(\mathrm{TR} 4 \Delta s p x B+$ ) restored the percentage of dead cells to nearly that of A549 cells treated with T4R supernatant (70 \% vs. $89 \%$, respectively). Similar results were obtained using a standard hemolysis assay, with supernatant from strains expressing PLY and SpxB leading to greater hemolysis than T4R $\Delta s p x B$ (Fig. 1b) Strains lacking both SpxB and PLY had no hemolysis, comparable to T4R $\Delta p l y$ (data not shown). These data demonstrate that expression of SpxB contributes to PLY-dependent cytotoxicity at mid-log phase growth.

SpxB is responsible for the production of $\mathrm{H}_{2} \mathrm{O}_{2}$ among $\mathrm{S}$. pneumoniae isolates at mid-log phase

S. pneumoniae is known to produce up to millimolar concentrations of $\mathrm{H}_{2} \mathrm{O}_{2}$ during growth [18]. The enzyme
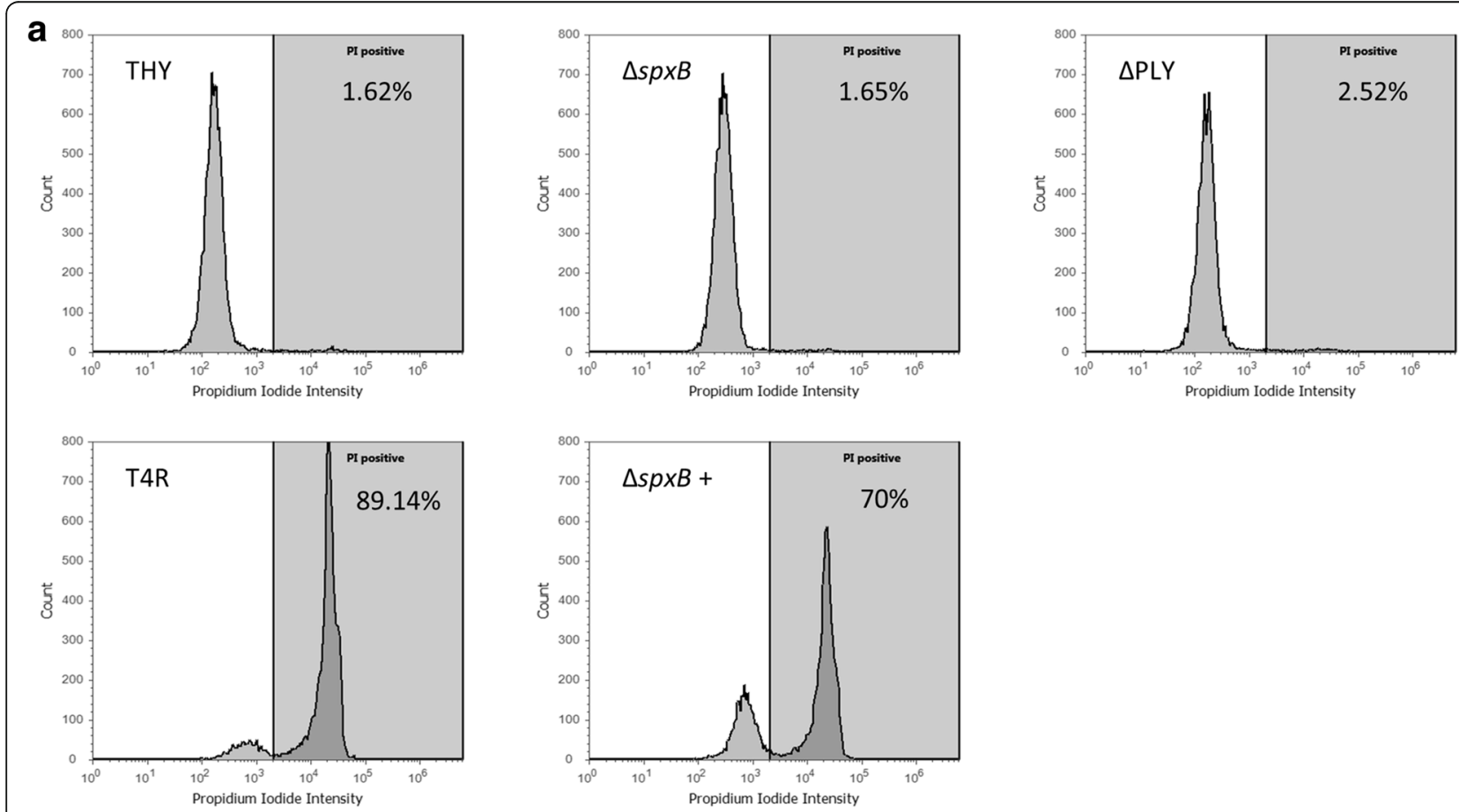

b

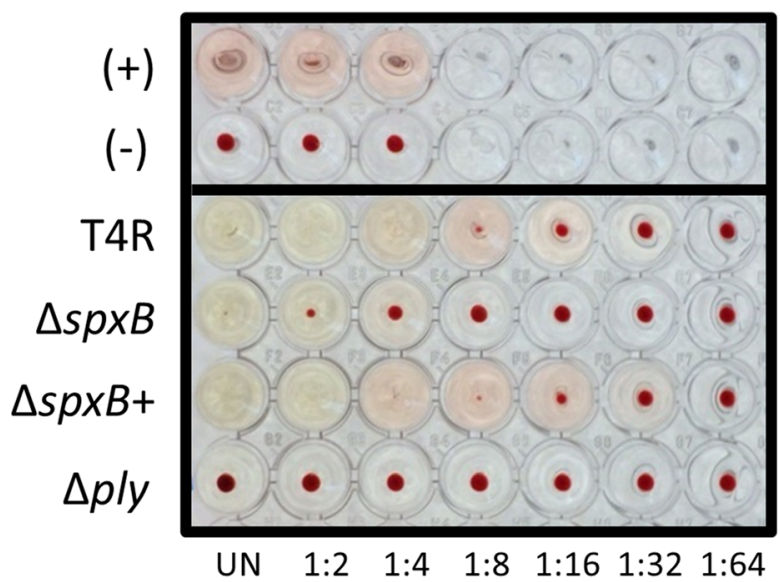

Fig. 1 Loss of SpxB reduces cytotoxtic potential of culture supernatants. a A549 human epithelial cells were exposed to culture supernatants from parental strain T4R and mutant strains lacking SpxB or PLY. Cytotoxicity was assesed by measuring propidium iodide uptake using flow cytometry. $\mathbf{b}$ Hemolytic effect of PLY released in culture supernatant. Serially diluted culture supernatants from T4R and mutant strains lacking SpxB were incubated with washed sheep red blood cells for $30 \mathrm{~min}$ and subsequently centrifuged. The absence of a red pellet at the bottom of well indicates lysis of RBCs. (+) indicates distilled water positive control for complete hemolysis and (-) received no PLY. Results are representative of duplicate experiments 
pyruvate oxidase $(\mathrm{SpxB})$ is known to be responsible for the majority of peroxide production by pneumococcus grown aerobically [21]. However, the enzyme lactate oxidase is also capable of producing $\mathrm{H}_{2} \mathrm{O}_{2}$ [33]. We assessed the relative production of $\mathrm{H}_{2} \mathrm{O}_{2}$ among a panel of laboratory strains. Pneumococcal strains T4, T4R, WU2, and AW267 and their isogenic $\triangle s p x B$ mutants were grown to mid-log phase $\left(\mathrm{OD}_{600}\right.$ of 0.5$)$ in THY and $\mathrm{H}_{2} \mathrm{O}_{2}$ was quantitated from filtered supernatants (Additional file 1: Figure S1 A-D). Peroxide concentrations were found to vary between strains. However, the deletion of $s p x B$ in all strains examined resulted in a significant reduction of $\mathrm{H}_{2} \mathrm{O}_{2}(P<0.005)$, which was comparable to supernatants from cultures grown in the presence of catalase. These results demonstrated that $\mathrm{SpxB}$ is primarily responsible for the production of the $\mathrm{H}_{2} \mathrm{O}_{2}$ by the strains examined in this study during mid-log phase growth.

SpxB enhances PLY release among S. pneumoniae isolates To determine the contribution of SpxB upon PLY release, supernatants from TIGR4, T4R, WU2, and AW267, and their isogenic $\triangle s p x B$ mutants, grown to $\mathrm{OD}_{600}$ of 0.5 , were analyzed by SDS-PAGE and western blot. A significant reduction in the amount of PLY released into the supernatants was observed in the $\triangle s p x B$ mutant of each strain examined (Fig. 2 a-d). Differences in PLY release between wild-type and $\triangle s p x B$ strains were not due to differences in colony forming units in the mid-log cultures as determined by plate counts (Additional file 2: Figure S2). Interestingly, addition of catalase to the medium only attenuated PLY release in high-releasing strains AW267 and WU2. Since catalase cannot cross the cell membrane and therefore can only neutralize extracellular $\mathrm{H}_{2} \mathrm{O}_{2}$, it is possible that the strains have different sensitivity to endogenous versus exogenous $\mathrm{H}_{2} \mathrm{O}_{2}$. Complementation of $s p \times B$ restored the ability of strains to release significant concentrations of PLY (Fig. 2 e and f).

To determine if PLY release correlated with $\mathrm{H}_{2} \mathrm{O}_{2}$ production in additional strains, a panel of 15 clinical isolates was analyzed. Each strain was grown to mid-log phase $\left(\mathrm{OD}_{600}\right.$ of 0.5), serial dilutions of the culture were plated, and supernatants were used for and PLY and $\mathrm{H}_{2} \mathrm{O}_{2}$ quantitation. A dot blot assay was used to quantitate PLY as plotted against known concentrations of recombinant PLY. PLY and $\mathrm{H}_{2} \mathrm{O}_{2}$ concentrations were normalized to 100,000 cells and a linear scatter plot was generated (Fig. 3). A significant correlation was observed between $\mathrm{H}_{2} \mathrm{O}_{2}$ production and PLY released among the isolates examined $\left(r^{2}=\right.$ $0.3167 ; p<0.05$ ). This suggests that $\mathrm{H}_{2} \mathrm{O}_{2}$ production impacts PLY release in clinically-relevant strains.

\section{Effects of SpxB on ply gene expression}

It is possible that expression of SpxB affects ply gene expression by metabolically altering the intracellular environment. To determine if deletion of $\operatorname{spx} B$ has a deleterious effect on ply gene transcription, leading to reduced amounts of PLY in the supernatant, qRT-PCR was performed on RNA isolated from strains grown to mid-log phase (Fig. 4). Surprisingly, the transcription of $p l y$ was found to be increased in all $\triangle s p x B$ mutants analyzed (AW267 $\Delta s p x B$ : 2.83-fold, WU2 $\Delta s p x B$ : 1.96-fold, T4R $\triangle$ spxB: 2.3-fold) which was in contrast to PLY release results shown in Fig. 2. Therefore, the reduced PLY release seen in $\triangle s p x B$ mutants is not due to reduced transcription of the ply gene.

\section{Impact of exogenous $\mathrm{H}_{2} \mathrm{O}_{2}$ on PLY release}

$\mathrm{H}_{2} \mathrm{O}_{2}$ is a by-product of SpxB activity and is known to have physiological effects upon pneumococcus. To determine the extent to which exogenous $\mathrm{H}_{2} \mathrm{O}_{2}$ can impact the release of PLY by inducing bactericidal lysis, $\mathrm{T} 4 \mathrm{R} \Delta \operatorname{sp} x B$ was treated with either $0 \mu \mathrm{M}$ or $500 \mu \mathrm{M}$ $\mathrm{H}_{2} \mathrm{O}_{2}$ on ice for $1 \mathrm{~h}$ and the concentration of PLY in the supernatant was determined via dot blot. Interestingly, no significant difference was found between the $\mathrm{H}_{2} \mathrm{O}_{2}$ treated bacteria and those not receiving $\mathrm{H}_{2} \mathrm{O}_{2}$ (Fig. 5a). To ensure exposure to $\mathrm{H}_{2} \mathrm{O}_{2}$ did not impact bacterial survival, and therefore PLY production, bacterial colony forming units were enumerated prior to and after $\mathrm{H}_{2} \mathrm{O}_{2}$ exposure. Concentrations up to $500 \mu \mathrm{M}$ did not impact bacterial survival (Additional file 3: Figure S3). Additionally, no significant difference in DNA release was seen with strains lacking $\operatorname{spxB}$ (Fig. 5b). These results, combined with the lack of catalase protection in the T4R strain (Fig. 2d), indicate that the contribution of SpxB to PLY release is not due to exogenous effects of $\mathrm{H}_{2} \mathrm{O}_{2}$ on bacterial viability and that $\mathrm{H}_{2} \mathrm{O}_{2}$ may exert its effects endogenously as it is being made.

\section{SpxB-dependent PLY release is not due to autolysis}

Another potential explanation for the difference in PLY release between the $\triangle s p \times B$ mutant and the parental strain is that loss of $\operatorname{spxB}$ leads to decreased autolysis even prior to stationary phase. Assays for autolysis were performed to determine if this was a contributing factor. Autolysis of S. pneumoniae involves the activity of the cell wall amidase LytA [34]. To determine whether LytA affected SpxB-dependent PLY release, western blots were performed on mid-log $\left(\mathrm{OD}_{600}\right.$ of 0.5) supernatants from parental strains $\mathrm{T} 4 \mathrm{R}$ and WU2 as well as isogenic mutants of both strains lacking either lytA or $s p x B$, or both (Fig. 6a). Mutants lacking $s p x B$, as expected, released less PLY, however loss of lytA had no significant effect on PLY release. These results indicate that the major autolysin, LytA, is not contributing to SpxBinduced PLY release at mid-log phase growth, but does not rule out its contribution at later times, such as during stationary phase. 
a

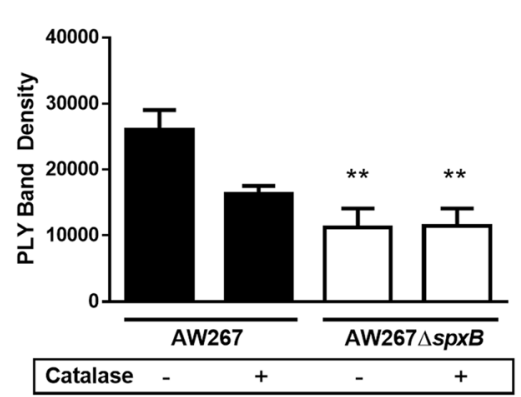

C
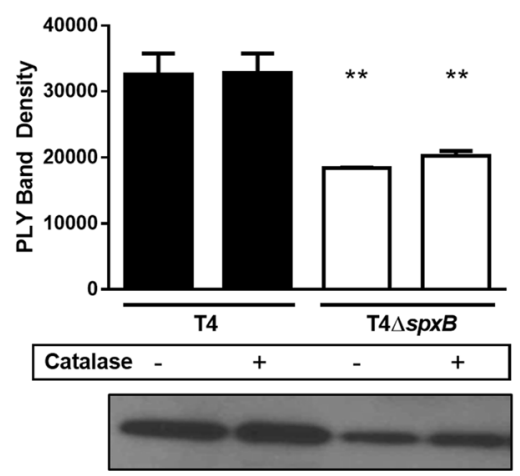

e

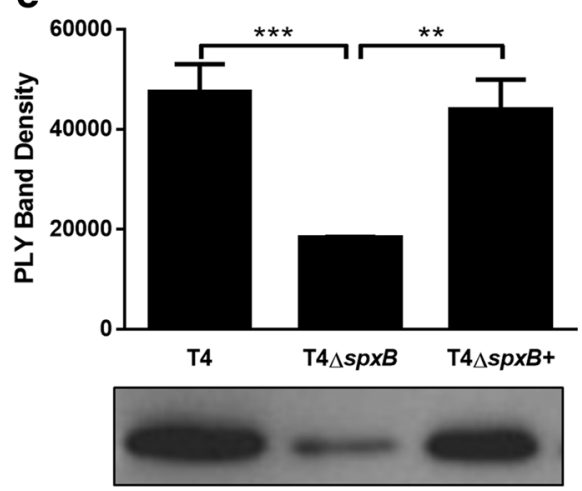

b

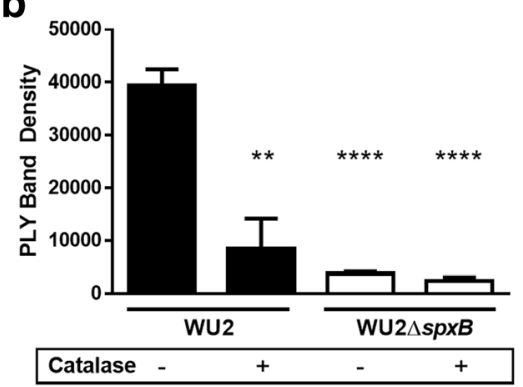

d
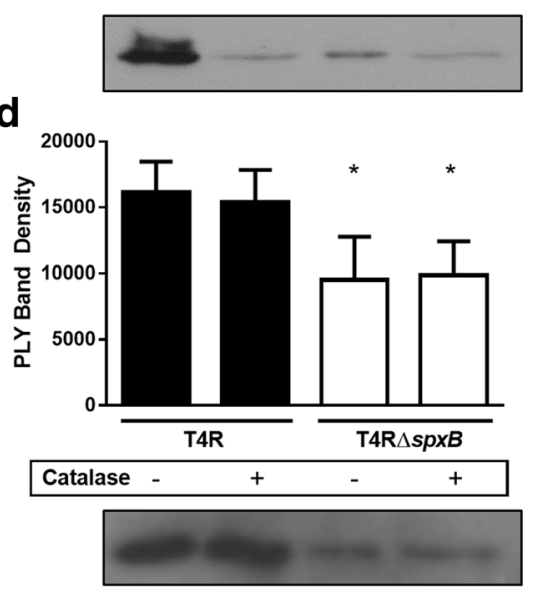

f

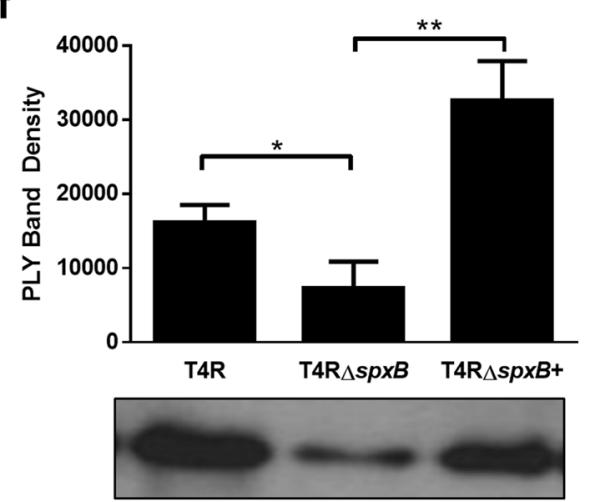

Fig. 2 Deletion of spXB greatly reduces PLY observed in the supernatant. a-d The amount of PLY released into culture supernatants from parental and mutant strains lacking SpxB (a AW267; b WU2, c T4, and $\mathbf{d}$ T4R) was measured by western blot. Band intensities were quantitated by densitometry. Results are representative of three independent experiments \pm SD. $\left({ }^{*} p<0.05,{ }^{* *} p<0.005,{ }^{* * *} p<0.00005\right)$. Where indicated, the cultures were complemented with $10 \mathrm{ng}$ of catalase. $\mathbf{e}$ and $\mathbf{f}$ The amount of PLY released in culture supernatants from parental, mutant strains lacking SpxB, and complemented strains (e T4; f T4R) was measured by western blot. Band intensities were quantitated by densitometry. Results are representative of three independent experiments \pm SD. ( ${ }^{*} p<0.05,{ }^{* *} p<0.005$, $\left.{ }^{* *} P<0.0005\right)$. Representative images of western blots are shown

It is possible that $\mathrm{H}_{2} \mathrm{O}_{2}$ or another by-product of SpxB activity could weaken the bacterial cell membrane, thereby facilitating PLY release. To determine if SpxB expression affected detergent-induced lysis, T4R and its isogenic $\triangle s p x B$ mutant were grown to an $\mathrm{OD}_{600}$ of 0.5 and then treated with the ionic detergent sodium deoxycholate (DOC). As expected, addition of DOC to midlog phase cultures initiated immediate lysis of both T4R and $\triangle \operatorname{spx} B$ strains. However, wild type T4R lysed more rapidly and to a greater extent than T4R $\Delta s p x B$. Supplementing the medium with catalase reduced lysis to levels comparable to T4R $\Delta s p x B$, indicating that $\mathrm{H}_{2} \mathrm{O}_{2}$ production may induce a condition that favors progression of lysis.

\section{Discussion}

Pneumolysin is a major virulence factor of pneumococcus and its release into the extracellular space has been shown to vary greatly between strains $[9,35]$. However, 


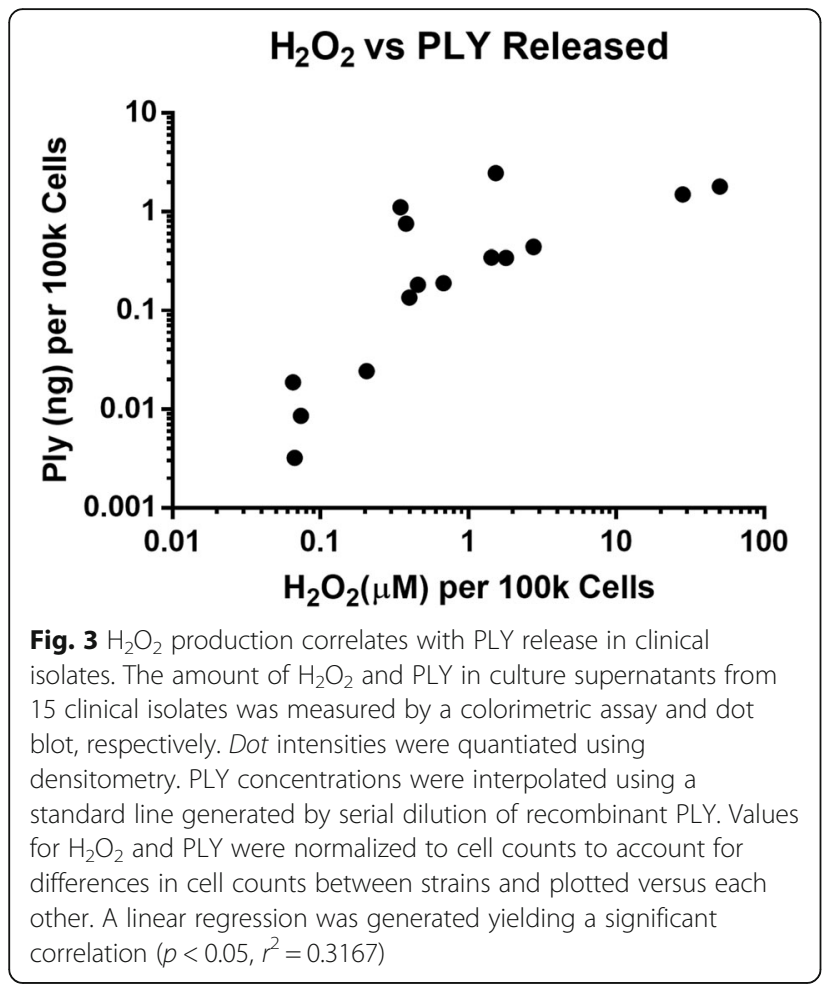

no single canonical mechanism of PLY release has been identified [8]. In this work, we have identified a novel link between expression of the metabolic enzyme pyruvate oxidase and release of PLY. Initially, we sought to determine the relative contribution of secreted $\mathrm{H}_{2} \mathrm{O}_{2}$ and PLY to host epithelial cytotoxicity. However, upon exposing A549 epithelial cells to pneumococcal supernatants, we found that supernatants from strains lacking
SpxB showed significantly decreased cytotoxicity, due to reduced extracellular PLY release. Since $\mathrm{H}_{2} \mathrm{O}_{2}$ has been shown to be the primary cause of pneumococcal autolysis during stationary phase [27], we initially suspected the loss of viability by $\mathrm{H}_{2} \mathrm{O}_{2}$ produced by $\mathrm{SpxB}$ might cause the enhanced release of PLY in culture supernatant. However, there was no significant difference in colony forming units between parental and $\operatorname{sp} x B \mathrm{mu}-$ tants. Additionally, there was no significant difference in extracellular DNA concentrations between parental and $\triangle s p x B$ mutants. Together, these results suggest that enhanced release of PLY in parental strains during mid-log phase growth was not due to a bactericidal effect of $\mathrm{H}_{2} \mathrm{O}_{2}$ produced by SpxB. While pneumococcal $\mathrm{H}_{2} \mathrm{O}_{2}$ is known to have cytotoxic effects on host cells $[24,26]$, our results indicate that the loss of host cell integrity is primarily linked to PLY released into the supernatant, not to $\mathrm{H}_{2} \mathrm{O}_{2}$. However, it is possible that SpxBdependent $\mathrm{H}_{2} \mathrm{O}_{2}$ induces stress within epithelial cells that may lead to alternative, non-cytolytic death processes. For instance, it has been shown that SpxBinduced $\mathrm{H}_{2} \mathrm{O}_{2}$ triggers genotoxicity and conserved stress responses within the epithelium $[24,36]$.

While $\mathrm{H}_{2} \mathrm{O}_{2}$-induced bactericidal effects do not appear to be the mechanism for PLY release, our findings do indicate that release may be linked to the production of $\mathrm{H}_{2} \mathrm{O}_{2}$, as indicated by the correlation between PLY release and $\mathrm{H}_{2} \mathrm{O}_{2}$ production in our clinical isolate panel. However, SpxB enzymatic activity produces other byproducts, including acetate and acetylphosphate that could potentially contribute to this mechanism of release [20]. Acetyl-phopshate contributes significantly to the ATP pool of pneumococcus [19]. We are currently
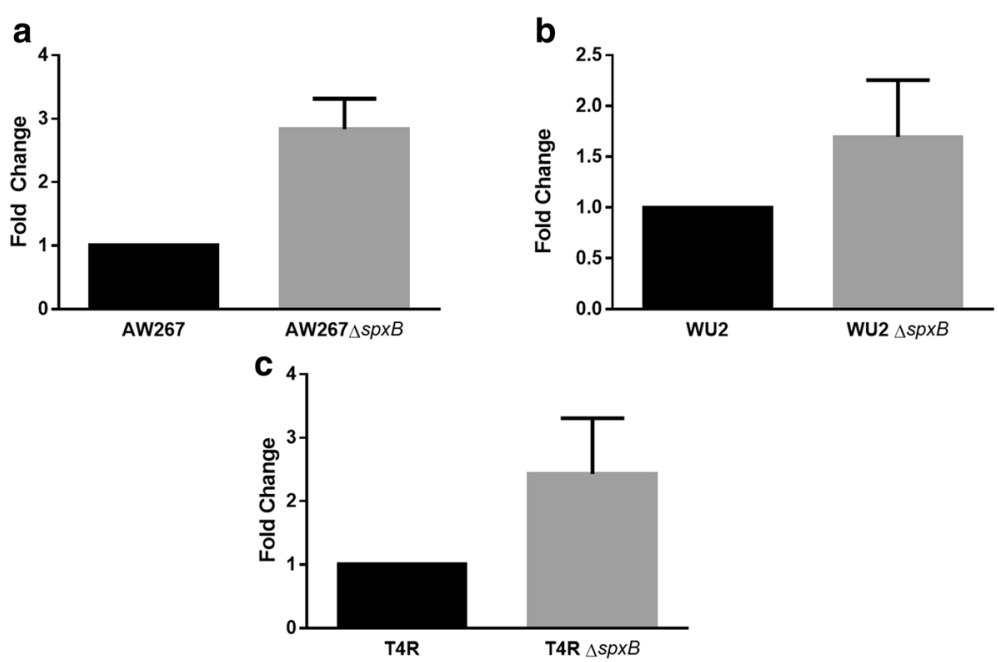

Fig. 4 SpxB-dependent PLY release is not due to decreased transcription of the ply gene. Strains AW267 (a), T4R (b), WU2 (c), and their respective $\triangle$ spxB mutants were grown to mid-log phase and RNA was isolated and used for CDNA synthesis followed by qRT-PCR. GyrA was utilized as an internal control housekeeping gene. Fold-changes were quantitated by the $2^{\Delta \Delta \mathrm{Ct}}$ method. Each figure represents three independent experiments \pm SD 

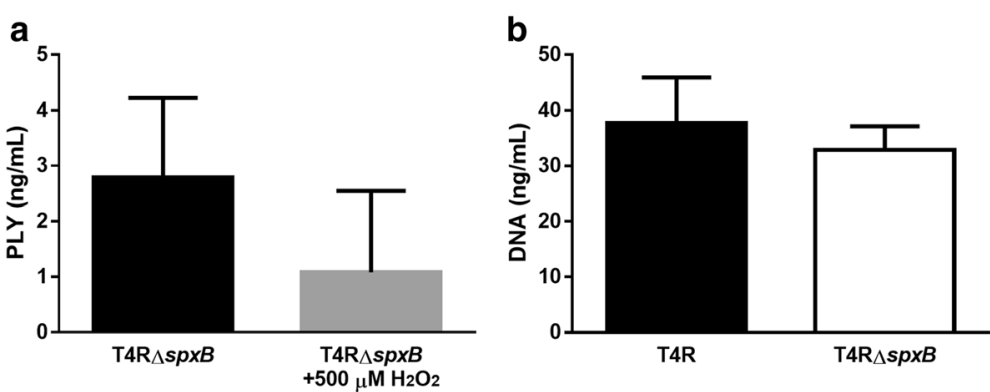

Fig. 5 Exogenous $\mathrm{H}_{2} \mathrm{O}_{2}$ does not induce PLY release. a The $\triangle S p x B$ mutant of T4R was grown to an OD of 0.5. The bacterial cells treated with $500 \mu \mathrm{M} \mathrm{H}_{2} \mathrm{O}_{2}$ for $1 \mathrm{~h}$ and PLY concentrations were quantitated via dot blot. Each figure represents three independent experiments \pm SD. $\mathbf{b}$ T4R and its $\triangle$ spxB mutant were grown to an $\mathrm{OD}_{600}$ of 0.5 and extracellular DNA was quantitated from sterile filtered supernatant by a Qbit fluorometer. Each figure represents three independent experiments \pm SD

investigating whether PLY release is energy-dependent. Furthermore, the addition of catalase to the culture medium abrogated PLY release from some strains (WU2), while failing to prevent release from others (TIGR4). These results suggest that strain-specific differences in metabolism might impact the release of PLY. We are also currently investigating how the capsule genes possessed by different serotypes may alter their metabolic processes and thereby $\mathrm{SpxB}$ activity and subsequent PLY release. However, if $\mathrm{H}_{2} \mathrm{O}_{2}$ serves to release PLY, it appears that it may act from within the bacterial cell, as supplementation of exogenous $\mathrm{H}_{2} \mathrm{O}_{2}$, surprisingly, did not enhance PLY release, at least in the T4R strain. Also, while both $s p x B$ and ply are known to be differentially expressed in different biological niches during infection [37], ply was not found to be expressed greater in parental strains as compared to $\triangle s p x B$ strains.

Our results indicate that the PLY release mechanism appears to be autolysis-independent, as mutants lacking the major autolytic cell wall amidase LytA were equally able to release PLY as parental strains. Furthermore, double mutants lacking both $\mathrm{SpxB}$ and LytA released

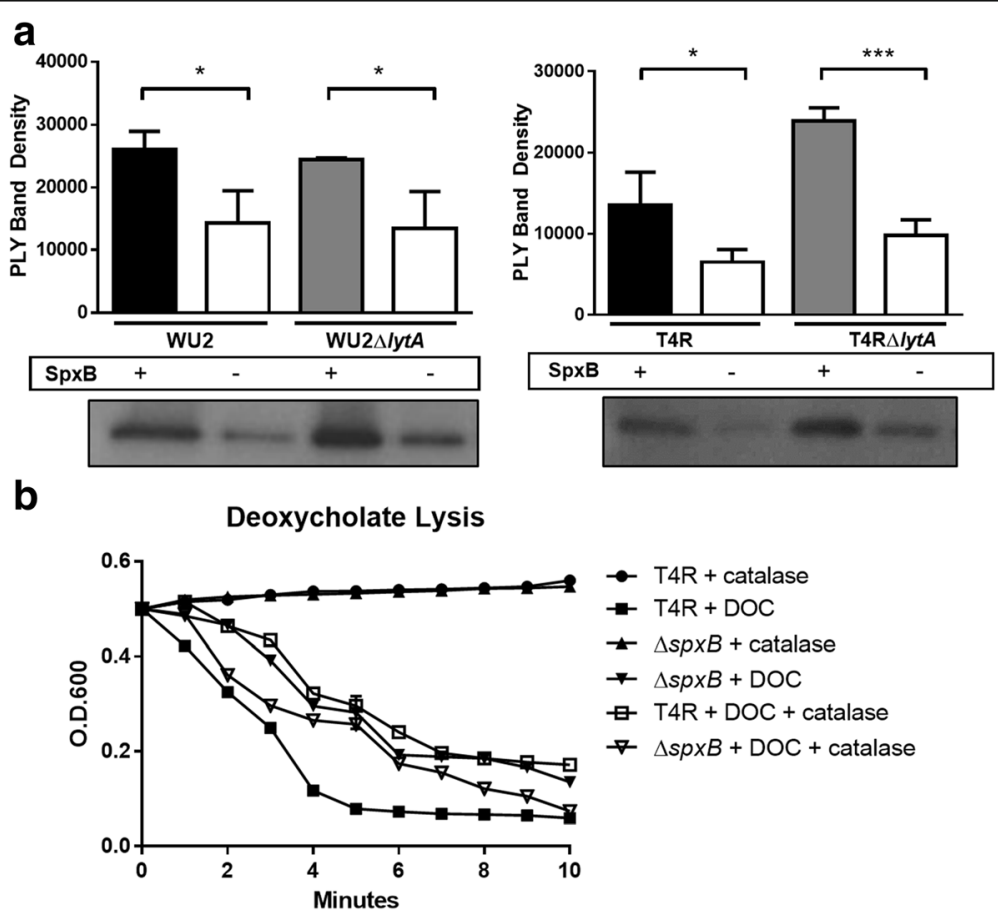

Fig. 6 Effects of SpxB upon detergent-induced lysis. a WU2 and T4R along with their $\triangle s p \times B, \triangle l y t A$, and $\triangle s p \times B / \triangle l y t A$ mutants were grown to an OD of 0.5. Equal volumes of sterile supernatants were run on SDS-PAGE gels and probed for PLY via western blot. Each figure represents three independent experiments \pm SD. $\left({ }^{*} p<0.05\right)$. A representative western blot image for each strain is shown. $\mathbf{b}$ T4R and its $\triangle s p \times B$ mutant were grown to an OD of 0.5 prior to treatment with $0.05 \%$ sodium deoxycholate (DOC). Spectrophotometric readings were taken every minute for 10 min to determine differences in the rate of induced autolysis 
equal PLY to strains lacking only SpxB. However, it is possible that additional autolytic factors including the murein hydrolase LytB, competence induced bacteriocin (CibAB), or choline binding protein $\mathrm{D}(\mathrm{CbpD})$ could play a role in sp $x B$-dependent PLY release [38-40]. Results from exposure of the parental strain T4R to deoxycholate indicate that $\operatorname{sp} x B$ expression may predispose the bacterial cells to autolysis. This indicates that $\mathrm{H}_{2} \mathrm{O}_{2}$ or some other SpxBinduced byproduct could weaken the bacterial cell membrane allowing for release of PLY from the intracellular space, possibly in the absence of cell death. However, loss of cell membrane integrity would likely free intracellular stores of LytA which could trigger autolytic effects not seen in our studies [41, 42]. An intriguing possibility is that PLY possesses characteristics that allow it to preferentially traverse the SpxB-perturbed membrane. We are currently investigating whether other cryptically secreted proteins may escape the cytosol via the SpxB-dependent mechanism used by PLY.

We have yet to determine is whether or not $\mathrm{H}_{2} \mathrm{O}_{2}$ produced by SpxB impacts pneumococcal activators or repressors, thereby indirectly affecting expression of genes that could impact PLY release. In Staphylococcus aureus, exposure to exogenous $\mathrm{H}_{2} \mathrm{O}_{2}$ enhanced expression of multiple oxidative stress response genes controlled by the ferric uptake regulator (Fur) homolog PerR [43]. While pneumococcus doesn't possess PerR, the iron uptake regulator RitR regulates expression of a number of genes which are impacted by both iron availability and oxidative stress [44]. The MerR regulators of pneumococcus are another group of regulatory proteins that can be impacted by oxidative stress [45].

Since SpxB is an important metabolic enzyme for the organism during aerobic growth, our findings could link cytotoxic potential of strains to metabolic shifts that occur at different niches throughout the host during colonization or progression to invasive disease. This mechanism could constitute another reason for the attenuated virulence seen with $s p x B$-negative mutants [21]. However, it was recently demonstrated that increased PLY release does not necessarily translate to enhanced virulence [16]. This underlines $\mathrm{SpxB}$ as an important virulence regulator, as our data indicate that SpxB expression contributes significantly to the release of PLY at early phases of growth, along with previous data indicating that it allows the organism to outcompete other common inhabitants of the nasopharynx [22]. These findings could represent a novel method for protein secretion that extends beyond pneumococcus to other bacterial species.

\section{Conclusions}

Expression of SpxB was shown to contribute to PLY release in various $S$. pneumoniae strains. While a panel of clinical isolates demonstrated a correlation between $\mathrm{H}_{2} \mathrm{O}_{2}$ production and PLY release, catalase was able to prevent PLY release in a strain-dependent manner. This indicates endogenous/exogenous $\mathrm{H}_{2} \mathrm{O}_{2}$ may contribute in a straindependent fashion to SpxB-dependent PLY release. PLY release due to $\operatorname{SpxB}$ was not dependent upon cellular turnover based on plate counts and DNA release. Though SpxB-dependent PLY release was not dependent on the activity of the cell wall amidase LytA, deoxycholateinduced autolysis was greater in strains expressing SpxB, indicating possible weakening of the cell membrane when $\mathrm{SpxB}$ is expressed. These results identify a novel route of PLY release that may extend to secretion of other cytoplasmic proteins that lack signal sequences.

\section{Additional files}

Additional file 1: Figure S1. Deletion of spxB greatly reduces production of hydrogen peroxide. The amount of hydrogen peroxide in culture supernatants from wild type and mutant strains lacking SpxB (A. AW267; B. WU2; C. T4, D. T4R) was measured via a colorimetric peroxide assay. Asterisks indicate statistical significance $\left.{ }^{* * * *} p<0.00005,{ }^{* *} p<0.005\right)$ and ND indicates a peroxide concentration below the detectable limits of the assay. (TIF $534 \mathrm{~kb}$ )

Additional file 2: Figure S2. Bacterial colony counts. Serial dilution plate counts were made at OD 0.5 of the indicated strains to determine if there was a difference in the bacterial counts when SpxB is removed, or when catalase is added. Each figure represents three independent experiments \pm SD. (TIF $540 \mathrm{~kb}$ )

Additional file 3: Figure S3. Bacterial colony counts. Serial dilution plate counts were made from cultures grown to $\mathrm{OD}_{600} 0.5$ prior to and $1 \mathrm{~h}$ post-exposure to various concentrations of $\mathrm{H}_{2} \mathrm{O}_{2}$. Results are shown as the average of 3 independent experiments \pm SD. (TIF $481 \mathrm{~kb}$ )

\section{Abbreviations}

$\mathrm{H}_{2} \mathrm{O}_{2}$ : Hydrogen peroxide; LytA: Autolysin; PLY: Pneumolysin; SpxB: Pyruvate oxidase

\section{Acknowledgements}

We would like to thank Mary Marquart for providing rabbit polyclonal antiPLY antibody.

\section{Funding}

JAT's lab is supported by an Institutional Development Award (IDeA) from the NIGMS COBRE grant number (P20GM103646), the MSU Department of Biological Sciences, and the MSU Office of Research and Economic Development.

\section{Availability of data and materials}

All materials used or generated in this study, including genetically modified strains, will be made freely available to any scientist wishing to use them for non-commercial purposes.

\section{Authors' contributions}

JB participated in design and completion of all experiments in the study and drafted the manuscript. RD performed autolysis assays and western blots. KO and LB performed molecular genetic studies and generated knockout strains. $J R, K S$, JD, and LM made substantial contributions to the conception and design of experiments. JT designed and coordinated all studies and contributed to drafting the manuscript. All authors read and approved the final manuscript.

Competing interests

The authors declare that they have no competing interests. 


\section{Consent for publication}

N/A.

\section{Ethics approval and consent to participate}

N/A

\section{Author details}

'Department of Biological Sciences, Mississippi State University, 295 E Lee Blvd., Harned Hall, Rm 219, Mississippi State, MS 39762, USA. ${ }^{2}$ Department of Infectious Diseases, St. Jude Children's Research Hospital, Memphis, TN, USA. ${ }^{3}$ Department of Basic Sciences, College of Veterinary Medicine, Mississipp State University, Mississippi State, MS, USA. ${ }^{4}$ Department of Biological Sciences, University of Southern Mississippi, Hattiesburg, MS, USA. ${ }^{5}$ Department of Microbiology and Immunology, University of Mississippi Medical Center, Jackson, MS, USA.

Received: 8 June 2016 Accepted: 28 October 2016 Published online: 09 November 2016

\section{References}

1. Bogaert D, De Groot R, Hermans PW. Streptococcus pneumoniae colonisation: the key to pneumococcal disease. Lancet Infect Dis. 2004;4(3):144-54.

2. O'Brien KL, Wolfson L, Watt JP, Henkle E, Deloria-Knoll M, McCall N, Lee E, Mulholland K, Levine OS, Cherian T. Burden of disease caused by Streptococcus pneumoniae in children younger than 5 years: global estimates. Lancet. 2009;374(9693):893-902.

3. Marcy M, Takata G, Chan LS, Shekelle P, Mason W, Wachsman L, Ernst R, Hay JW, Corley PM, Morphew T, et al. Management of acute otitis media. Evid Rep Technol Assess (Summ). 2000;15:1-4.

4. Le Polain de Waroux O, Flasche S, Prieto-Merino D, Edmunds WJ. Agedependent prevalence of nasopharyngeal carriage of streptococcus pneumoniae before conjugate vaccine introduction: a prediction model based on a meta-analysis. PLoS One. 2014;9(1):e86136.

5. Berry AM, Lock RA, Thomas SM, Rajan DP, Hansman D, Paton JC. Cloning and nucleotide sequence of the Streptococcus pneumoniae hyaluronidase gene and purification of the enzyme from recombinant Escherichia coli. Infect Immun. 1994;62(3):1101-8.

6. Briles DE, Yother J, McDaniel LS. Role of pneumococcal surface protein A in the virulence of Streptococcus pneumoniae. Rev Infect Dis. 1988;10 Suppl 2:S372-4.

7. Camara M, Boulnois GJ, Andrew PW, Mitchell TJ. A neuraminidase from Streptococcus pneumoniae has the features of a surface protein. Infect Immun. 1994:62(9):3688-95.

8. Mitchell TJ, Dalziel CE. The biology of pneumolysin. Subcell Biochem. 2014;80:145-60.

9. Marriott HM, Jackson LE, Wilkinson TS, Simpson AJ, Mitchell TJ, Buttle DJ, Cross SS, Ince PG, Hellewell PG, Whyte MK, et al. Reactive oxygen species regulate neutrophil recruitment and survival in pneumococcal pneumonia. Am J Respir Crit Care Med. 2008;177(8):887-95.

10. Rogers PD, Thornton J, Barker KS, McDaniel DO, Sacks GS, Swiatlo E, McDanie LS. Pneumolysin-dependent and -independent gene expression identified by CDNA microarray analysis of THP-1 human mononuclear cells stimulated by Streptococcus pneumoniae. Infect Immun. 2003;71(4):2087-94.

11. Walker JA, Allen RL, Falmagne P, Johnson MK, Boulnois GJ. Molecular cloning, characterization, and complete nucleotide sequence of the gene for pneumolysin, the sulfhydryl-activated toxin of Streptococcus pneumoniae. Infect Immun. 1987;55(5):1184-9.

12. Price $K E$, Greene NG, Camilli A. Export requirements of pneumolysin in Streptococcus pneumoniae. J Bacteriol. 2012;194(14):3651-60.

13. Mitchell TJ, Alexander JE, Morgan PJ, Andrew PW. Molecular analysis of virulence factors of Streptococcus pneumoniae. Soc Appl Bacteriol Symp Ser. 1997;26:62S-71.

14. Balachandran P, Hollingshead SK, Paton JC, Briles DE. The autolytic enzyme LytA of Streptococcus pneumoniae is not responsible for releasing pneumolysin. J Bacteriol. 2001;183(10):3108-16.

15. Price KE, Camilli A. Pneumolysin localizes to the cell wall of Streptococcus pneumoniae. J Bacteriol. 2009;191(7):2163-8.

16. Greene NG, Narciso AR, Filipe SR, Camilli A. Peptidoglycan Branched Stem Peptides Contribute to Streptococcus pneumoniae Virulence by Inhibiting Pneumolysin Release. PLoS Pathog. 2015;11(6):e1004996.
17. Marriott HM, Mitchell TJ, Dockrell DH. Pneumolysin: a double-edged sword during the host-pathogen interaction. Curr Mol Med. 2008:8(6):497-509.

18. McLeod JW, Gordon J. Production of Hydrogen Peroxide by Bacteria. Biochem J. 1922;16(4):499-506.

19. Pericone CD, Park S, Imlay JA, Weiser JN. Factors contributing to hydrogen peroxide resistance in Streptococcus pneumoniae include pyruvate oxidase (SpxB) and avoidance of the toxic effects of the fenton reaction. J Bacteriol. 2003;185(23):6815-25

20. Taniai H, lida K, Seki M, Saito M, Shiota S, Nakayama H, Yoshida S. Concerted action of lactate oxidase and pyruvate oxidase in aerobic growth of Streptococcus pneumoniae: role of lactate as an energy source. J Bacteriol. 2008;190(10):3572-9.

21. Spellerberg B, Cundell DR, Sandros J, Pearce BJ, Idanpaan-Heikkila I, Rosenow C, Masure HR. Pyruvate oxidase, as a determinant of virulence in Streptococcus pneumoniae. Mol Microbiol. 1996;19(4):803-13.

22. Pericone CD, Overweg K, Hermans PW, Weiser JN. Inhibitory and bactericidal effects of hydrogen peroxide production by Streptococcus pneumoniae on other inhabitants of the upper respiratory tract. Infect Immun. 2000;68(7):3990-7.

23. Braun JS, Sublett JE, Freyer D, Mitchell TJ, Cleveland JL, Tuomanen El, Weber JR. Pneumococcal pneumolysin and $\mathrm{H}(2) \mathrm{O}(2)$ mediate brain cell apoptosis during meningitis. J Clin Invest. 2002;109(1):19-27.

24. Rai P, Parrish M, Tay IJ, Li N, Ackerman S, He F, Kwang J, Chow VT, Engelward BP. Streptococcus pneumoniae secretes hydrogen peroxide leading to DNA damage and apoptosis in lung cells. Proc Natl Acad Sci U S A. 2015;112(26):E3421-30.

25. Rubins JB, Duane PG, Charboneau D, Janoff EN. Toxicity of pneumolysin to pulmonary endothelial cells in vitro. Infect Immun. 1992;60(5):1740-6.

26. Zysk G, Bejo L, Schneider-Wald BK, Nau R, Heinz H. Induction of necrosis and apoptosis of neutrophil granulocytes by Streptococcus pneumoniae. Clin Exp Immunol. 2000;122(1):61-6.

27. Regev-Yochay G, Trzcinski K, Thompson CM, Lipsitch M, Malley R. SpxB is a suicide gene of Streptococcus pneumoniae and confers a selective advantage in an in vivo competitive colonization model. J Bacteriol. 2007;189(18):6532-9.

28. Pesakhov S, Benisty R, Sikron N, Cohen Z, Gomelsky P, Khozin-Goldberg I, Dagan R, Porat N. Effect of hydrogen peroxide production and the Fenton reaction on membrane composition of Streptococcus pneumoniae. Biochim Biophys Acta. 2007;1768(3):590-7.

29. Tettelin H, Nelson KE, Paulsen IT, Eisen JA, Read TD, Peterson S, Heidelberg J, DeBoy RT, Haft DH, Dodson RJ, et al. Complete genome sequence of a virulent isolate of Streptococcus pneumoniae. Science. 2001:293(5529):498-506.

30. Fernebro J, Andersson I, Sublett J, Morfeldt E, Novak R, Tuomanen E, Normark S, Normark BH. Capsular expression in Streptococcus pneumoniae negatively affects spontaneous and antibiotic-induced lysis and contributes to antibiotic tolerance. J Infect Dis. 2004:189(2):328-38.

31. Briles DE, Nahm M, Schroer K, Davie J, Baker P, Kearney J, Barletta R. Antiphosphocholine antibodies found in normal mouse serum are protective against intravenous infection with type 3 streptococcus pneumoniae. J Exp Med. 1981;153(3):694-705.

32. Thornton JA. Splicing by Overlap Extension PCR to Obtain Hybrid DNA Products. Methods Mol Biol. 2016;1373:43-9.

33. Udaka S, Koukol J, Vennesland B. Lactic oxidase of Pneumococcus. Bacteriol. 1959:78:714-25.

34. Ronda C, Garcia JL, Garcia E, Sanchez-Puelles JM, Lopez R. Biological role of the pneumococcal amidase. Cloning of the lytA gene in Streptococcus pneumoniae. Eur J Biochem. 1987;164(3):621-4.

35. Benton KA, Paton JC, Briles DE. Differences in virulence for mice among Streptococcus pneumoniae strains of capsular types 2, 3, 4, 5, and 6 are not attributable to differences in pneumolysin production. Infect Immun. 1997:65(4):1237-44.

36. Loose M, Hudel M, Zimmer KP, Garcia E, Hammerschmidt S, Lucas R, Chakraborty T, Pillich H. Pneumococcal hydrogen peroxide-induced stress signaling regulates inflammatory genes. J Infect Dis. 2015;211(2):306-16.

37. Orihuela CJ, Radin JN, Sublett JE, Gao G, Kaushal D, Tuomanen El. Microarray analysis of pneumococcal gene expression during invasive disease. Infect Immun. 2004:72(10):5582-96.

38. Garcia P, Gonzalez MP, Garcia E, Lopez R, Garcia JL. LytB, a novel pneumococcal murein hydrolase essential for cell separation. Mol Microbiol. 1999;31(4):1275-81. 
39. Kausmally L, Johnsborg O, Lunde M, Knutsen E, Havarstein LS. Cholinebinding protein $\mathrm{D}(\mathrm{CbpD})$ in Streptococcus pneumoniae is essential for competence-induced cell lysis. J Bacteriol. 2005;187(13):4338-45.

40. Guiral S, Mitchell TJ, Martin B, Claverys JP. Competence-programmed predation of noncompetent cells in the human pathogen Streptococcus pneumoniae: genetic requirements. Proc Natl Acad Sci U S A. 2005;102(24):8710-5.

41. Mellroth P, Sandalova T, Kikhney A, Vilaplana F, Hesek D, Lee M, Mobashery S, Normark S, Svergun D, Henriques-Normark B, et al. Structural and functional insights into peptidoglycan access for the lytic amidase LytA of Streptococcus pneumoniae. MBio. 2014;5(1):e01120-01113.

42. Mellroth $P$, Daniels R, Eberhardt A, Ronnlund D, Blom H, Widengren J, Normark S, Henriques-Normark B. LytA, major autolysin of Streptococcus pneumoniae, requires access to nascent peptidoglycan. J Biol Chem. 2012:287(14):11018-29.

43. Horsburgh MJ, Clements MO, Crossley H, Ingham E, Foster SJ. PerR controls oxidative stress resistance and iron storage proteins and is required for virulence in Staphylococcus aureus. Infect Immun. 2001;69(6):3744-54.

44. Ulijasz AT, Andes DR, Glasner JD, Weisblum B. Regulation of iron transport in Streptococcus pneumoniae by RitR, an orphan response regulator. J Bacteriol. 2004;186(23):8123-36.

45. Brown NL, Stoyanov JV, Kidd SP, Hobman JL. The MerR family of transcriptional regulators. FEMS Microbiol Rev. 2003;27(2-3):145-63.

\section{Submit your next manuscript to BioMed Central} and we will help you at every step:

- We accept pre-submission inquiries

- Our selector tool helps you to find the most relevant journal

- We provide round the clock customer support

- Convenient online submission

- Thorough peer review

- Inclusion in PubMed and all major indexing services

- Maximum visibility for your research

Submit your manuscript at www.biomedcentral.com/submit 